\title{
Desigualdad en el Ingreso, Corrupción y Esperanza de Vida al Nacer en México
}

Alvaro Javier Idrovo

Médico, Especialista en Higiene y Salud Ocupacional, M. Sc. Salud Pública, M. Sc. Salud Ambiental, Ph. D (Candidato) en Epidemiología. Centro de Investigación en Salud Poblacional, Instituto Nacional de Salud Pública. Cuernavaca, Morelos, México.

E-mail: idrovoaj@hotmail.com,idrovoaj@yahoo.com.mx

Recibido 14 Abril 2005/Enviado Modificación 15 Mayo 2005/Aceptado 2 Junio 2005

\section{RESUMEN}

Objetivo Indagar si el efecto de la desigualdad en el ingreso sobre la esperanza de vida al nacer en México está mediado por la corrupción, usada como proxy del capital social.

Material y Métodos Con las 32 entidades federativas mexicanas se realizó un estudio ecológico. Se estimaron las correlaciones entre la esperanza de vida al nacer, total y por sexo, y el coeficiente de Gini, el Indice de Corrupción y Buen Gobierno, el porcentaje de católicos y el porcentaje de población que habla lengua indígena por entidad federativa. Regresiones lineales robustas, con y sin variables instrumentales, fueron usadas para explorar si la corrupción actúa como variable intermedia en la relación estudiada.

Resultados Se observaron correlaciones negativas con rho de Spearman cercanas a $-0.60(p<0.05)$ y superiores a $-0.66(p<0.05)$ entre la esperanza de vida al nacer y el coeficiente de Gini y el porcentaje de la población que habla lengua indígena, respectivamente. El Indice de Corrupción y Buen Gobierno, además, se correlacionó con la esperanza de vida al nacer de los hombres con una rho de Spearman de $-0.3592(p<0.05)$. Las regresiones con instrumentos resultaron ser más consistentes que las convencionales, y mostraron un fuerte efecto negativo $(p<0.05)$ de la desigualdad en el ingreso sobre la esperanza de vida al nacer. Este efecto resulta ser mayor entre los hombres.

Discusión Los resultados sugieren un efecto negativo de la desigualdad en el ingreso sobre la esperanza de vida al nacer en México, mediado por los niveles de corrupción y otros factores culturales asociados.

Palabras Clave: Clase social, pobreza, distribución espacial, México (fuente: DeCS, BIREME). 


\begin{abstract}
Income inequality, corruption, and life expectancy at birth in Mexico

Objective To ascertain if the effect of income inequality on life expectancy at birth in Mexico is mediated by corruption, used as a proxy of social capital.

Material and Methods An ecological study was carried out with the 32 Mexican federative entities. Global and by sex correlations between life expectancy at birth were estimated by federative entity with the Gini coefficient, the Corruption and Good Government Index, the percentage of Catholics, and the percentage of the population speaking indigenous language. Robust linear regressions, with and without instrumental variables, were used to explore if corruption acts as intermediate variable in the studied relationship.

Results Negative correlations with Spearman's rho near to $-0.60 \quad(p<0.05)$ and greater than $-0.66(p<0.05)$ between life expectancy at birth, the Gini coefficient and the population speaking indigenous language, respectively, were observed. Moreover, the Corruption and Good Government Index correlated with men's life expectancy at birth with Spearman's rho -0.3592 $(p<0.05)$. Regressions with instruments were more consistent than conventional ones and they show a strong negative effect $(p<0.05)$ of income inequality on life expectancy at birth. This effect was greater among men.

Conclusion The findings suggest a negative effect of income inequality on life expectancy at birth in Mexico, mediated by corruption levels and other related cultural factors.
\end{abstract}

Key Words: Social class, poverty, residence characteristics; Mexico (source: $\mathrm{MeSH}, \mathrm{NLM}$ ).

$\mathrm{E}$ l impacto negativo de la pobreza sobre la salud no tiene discusión en la actualidad (1). En las últimas décadas, la epidemiología social ha superado la dicotomía entre ricos y pobres, y ha puesto en evidencia que existe un efecto de la desigualdad en el ingreso, incluso dentro de poblaciones no pobres, sobre la salud poblacional e individual (2-4). Los mecanismos involucrados en esta relación aún no son muy conocidos, aunque existen algunas evidencias que señalan al capital social como variable intermedia (5). El capital social ha sido definido de múltiples maneras, y se puede afirmar que está constituido por características de la organización social tales como las redes de asociaciones secundarias, los altos niveles de confianza entre las personas y las normas de mutua ayuda y reciprocidad, las cuales actúan como fuentes para los individuos y facilitan la acción colectiva (6). Si bien existen otras propuestas de definición, los atributos comunes a todas estas son que: $i$ ) es un constructo que se puede medir a nivel poblacional y no individual, dado que es una característica de las sociedades y ii) es un bien público, de manera que no excluye a ninguno de los miembros de una sociedad (7). 
Bjørnskov (8), revisando las formas de medición identificó tres tipos de indicadores de capital social: i) la confianza generalizada, el cual es un indicador directo que suele ser medido como el porcentaje de respuestas positivas a preguntas sobre el grado de confianza que se puede tener en la gente, ii) la corrupción percibida, el cual es un indicador indirecto, y que puede ser medida mediante encuestas a expertos o el público en general, y iii) la participación cívica, el cual suele medirse mediante el porcentaje de individuos miembros de organizaciones cívicas voluntarias (9). Al comparar estos indicadores, algunos estudios empíricos sugieren que el nivel de corrupción puede ser superior a los otros dos $(8,10)$. Pese a estas posibilidades de medición, una de las formas más comunes de medir el capital social en epidemiología es mediante las tasas de crimen violento (6), debido a la mayor disponibilidad de estos datos. El uso de las tasas de crimen violento se sustenta en la teoría de la desorganización social, originaria de la criminología, que señala que la capacidad de un vecindario de controlar el crimen depende del control social informal; es decir, del deseo de los residentes de intervenir a nombre de todos buscando un bien común, lo cual implica una base de confianza y solidaridad mutua (11). Dadas las limitaciones que esta forma de medición ha mostrado en estudios empíricos (12), en este artículo se explora el efecto contextual potencial de la desigualdad en el ingreso sobre la salud poblacional, teniendo al capital social como variable intermedia en esta cadena causal, y medida mediante un índice de la corrupción percibida. Es importante aclarar que el concepto de corrupción ha variado con el paso del tiempo, aunque para fines prácticos resulta muy útil usar la definición amplia de McMullan; éste autor afirma que existe corrupción cuando un individuo o un grupo acepta dinero o un pago en especie por hacer alguna cosa que tiene el deber o no de hacerla, o de ejercer una discreción legítima por razones impropias (13).

Este estudio se realiza con datos de México, país en el cual un alto porcentaje de la población está en condiciones de pobreza o extrema pobreza (14), y aún no se ha determinado con certeza la magnitud del efecto de la desigualdad en el ingreso sobre la salud ni mucho menos las vías involucradas en dicha relación. Dados estos vacíos de conocimiento, el objetivo del presente estudio fue explorar si el efecto de la desigualdad en el ingreso sobre la esperanza de vida al nacer (EVN) en México está mediado por la corrupción. Debido a que la corrupción puede ser explicada por factores culturales, en este caso se utilizarán los porcentajes de la población que profesan religión católica (15) y de la población que habla lengua indígena como proxys de estos factores. La EVN es una variable composicional (16) que puede definirse como el número promedio de años adicionales que una per- 
sona podría vivir si las tendencias en la mortalidad actuales continuaran por el resto de su vida (17). Dado que la esperanza de vida es fuertemente dependiente de los criterios usados para seleccionar los grupos, en regiones con alta mortalidad infantil la EVN es altamente sensible a la muerte en los primeros años de vida.

\section{MATERIALES Y MÉTODOS}

Se realizó un estudio ecológico de múltiples grupos con las 32 entidades federativas mexicanas. De los registros del Instituto Nacional de Estadística, Geografía e Informática (INEGI) fueron obtenidas las EVN, total y por sexo, para 2003, el porcentaje de católicos mayores de cinco años de edad para 2000 y el porcentaje de población mayor de cinco años de edad que habla lengua indígena para 2000 de cada una de las entidades federativas. Los coeficientes de Gini utilizados fueron estimados por Rodolfo Tuirán Gutiérrez, reconocido economista mexicano, con datos de 2000 y se encuentran publicados en otro trabajo (18); este índice es la forma más frecuente de medir la desigualdad en el ingreso y presenta valores entre cero y uno, siendo mayor cuando más concentración de la riqueza hay y menor cuando es más igualitaria la distribución del ingreso (19). El nivel de corrupción fue estimado mediante el Índice de Corrupción y Buen Gobierno por entidad federativa (ICBGe) de 2001, elaborado por Transparencia Mexicana (20). Este índice se construyó teniendo como fuente las respuestas de 13790 hogares de todo el país sobre la corrupción en servicios públicos y privados; se consideró que hubo corrupción cuando el entrevistado refirió haber pagado a cambio de un servicio. Los valores posibles están entre cero y 100, siendo mayor entre más corrupción sea reportada. Más detalles sobre la construcción del índice y la encuesta pueden encontrarse en la página de Transparencia Mexicana (http://www.transparenciamexicana.org.mx).

\section{Análisis estadístico}

En un primer momento las correlaciones entre las variables dependientes e independientes fueron calculadas mediante pruebas de correlación de Spearman. Dado que existen algunas evidencias de que el capital social puede actuar como variable intermedia en la relación entre la desigualdad en el ingreso y salud poblacional (21), esta posibilidad fue explorada mediante el uso de regresiones robustas en las que la variable dependiente fue la EVN, total y por sexo, y el ICBGe, el porcentaje de católicos y el porcentaje de población que habla lengua indígena fueron instrumentos (22) del coeficiente de Gini. Adicionalmente se realizaron regresiones lineales múltiples 
robustas convencionales. La consistencia de los estimadores obtenidos mediante los dos métodos posteriormente fue evaluada con la prueba de Hausman (23). Todos estos análisis fueron realizados con el programa estadístico Stata 8 (Stata Corporation, College Station, Texas).

\section{RESULTADOS}

La EVN total para México fue de 74,9 años, con una desviación estándar (d.e.) de 0.75 , siendo mayor para las mujeres $(77,3$ años; d.e. 0,66$)$ y menor para los hombres (72,4 años; d.e. 0,85). En la Tabla 1 se pueden observar las correlaciones entre las variables dependientes y dependientes estudiadas. Allí se puede observar una correlación consistente entre la desigualdad en el ingreso, expresada con el coeficiente de Gini, y la EVN total y por sexo; algo similar ocurre con la población que habla lengua indígena. En ambos casos las correlaciones son mayores entre los hombres. Durante la exploración de las demás variables sólo se observó una correlación significativa entre el ICBGe y la EVN de hombres.

En la Figura 1 se aprecia la relación entre el coeficiente de Gini y la EVN total, la cual se puede expresar con la ecuación EVN total=$9.829 *$ coeficiente de Gini $+80.541\left(r^{2}=0.4404\right)$. En la Tabla 2 se resumen los modelos lineales robustos, con y sin variables instrumentales; allí nuevamente se observa que el coeficiente de Gini se encuentra relacionado con la EVN total y por sexo. Al evaluar la consistencia de los estimadores obtenidos, la prueba de Hausman mostró diferencias significativas $(p<0.05)$ en todos los casos, lo que apoya la idea de que los modelos lineales convencionales son estimadores inconsistentes de las ecuaciones.

Figura 1. Desigualdad en el ingreso y esperanza de vida al nacer en México por entidad federativa, 2003.

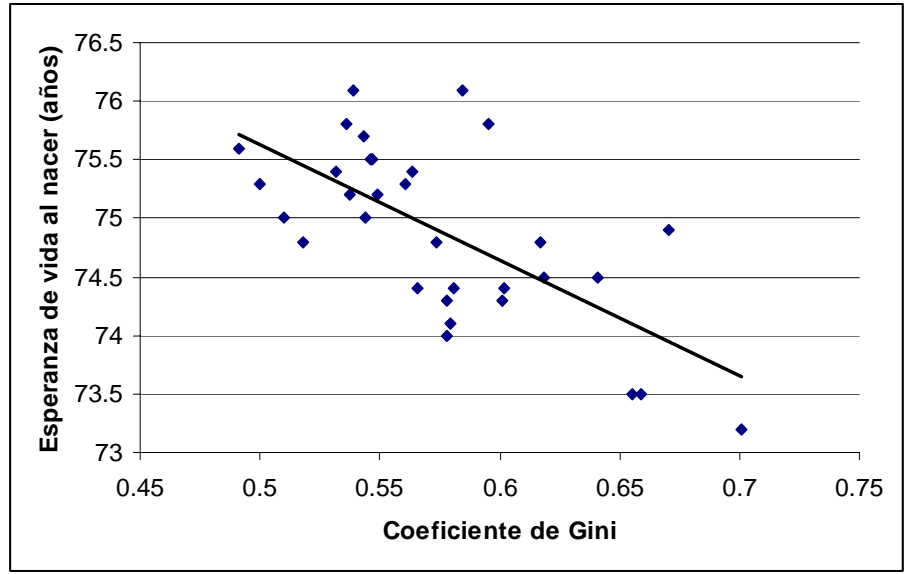


REVISTA DE SALUD PÚBLICA • Volumen 7 (2), Julio 2005

Tabla 1. Correlaciones de Spearman entre la esperanza de vida al nacer y los indicadores de desigualdad en el ingreso, nivel de corrupción y el porcentaje de católicos en las entidades federativas mexicanas.

\begin{tabular}{|c|c|c|c|c|}
\hline $\begin{array}{l}\text { Esperanza de } \\
\text { vida al nacer }\end{array}$ & $\begin{array}{l}\text { Coeficiente } \\
\text { De Gini }\end{array}$ & ICBGe & $\begin{array}{c}\text { Catolicismo } \\
(\%)\end{array}$ & $\begin{array}{c}\text { Población con } \\
\text { lengua } \\
\text { indígena (\%) } \\
\end{array}$ \\
\hline Total & $-0.6027^{*}$ & -0.3398 & 0.1295 & $-0.6901^{*}$ \\
\hline Hombres & $-0.6158^{*}$ & $0.3592^{*}$ & 0.1009 & $-0.6984^{*}$ \\
\hline Mujeres & $-0.6072^{\star}$ & -0.3181 & 0.1252 & $-0.6655^{\star}$ \\
\hline
\end{tabular}

Tabla 2. Relación entre esperanza de vida al nacer, total y por sexo, y la desigualdad en el ingreso, corrupción, catolicismo y población con lengua indígena en las entidades federativas mexicanas

\begin{tabular}{rlccccccc}
\hline \multirow{2}{*}{$\begin{array}{c}\text { Esperanza de } \\
\text { Vida al nacer }\end{array}$} & \multicolumn{2}{c}{ Coeficiente de Gini } & \multicolumn{2}{c}{ ICBGe } & \multicolumn{2}{c}{$\begin{array}{c}\text { Catolicismo } \\
(\%)\end{array}$} & \multicolumn{2}{c}{$\begin{array}{c}\text { Población con } \\
\text { lengua indígena (\%) }\end{array}$} \\
\cline { 2 - 9 } & $\beta$ & IC 95\% & $\beta$ & IC 95\% & $\beta$ & IC 95\% & $\beta$ & IC 95\% \\
\hline Total & & & & & & & & \\
Modelo 1 & -22.23 & $-36.94 ;-7.52$ & NA & & NA & & NA & \\
Modelo 2 & -7.38 & $-11.15 ;-3.62$ & 0.01 & $-0.04 ; 0.06$ & -0.01 & $-0.03 ; 0.02$ & -0.03 & $-0.06 ;-0.01$ \\
\hline Hombres & & & & & & & & \\
$\quad$ Modelo 1 & -25.02 & $-41.59 ;-8.46$ & NA & & NA & & NA & \\
Modelo 2 & -8.17 & $-12.42 ;-3.93$ & 0.01 & $-0.05 ; 0.07$ & -0.01 & $-0.04 ; 0.02$ & -0.04 & $-0.07 ;-0.02$ \\
\hline Mujeres & & & & & & & & \\
Modelo 1 & -19.13 & $-31.82 ;-6.45$ & NA & & NA & & NA & \\
Modelo 2 & -6.57 & $-9.95 ;-3.20$ & 0.02 & $-0.03 ; 0.06$ & -0.01 & $-0.03 ; 0.02$ & -0.03 & $-0.05 ;-0.01$ \\
\hline
\end{tabular}

ICBGe: Indice de Corrupción y Buen Gobierno por entidad federativa calculado por Transparencia Mexicana.

IC 95\%: intervalo de confianza de $95 \%$.

NA: No aplica; son variables que intervienen como instrumentos. 


\section{DISCUSIÓN}

El principal hallazgo de este estudio es que la desigualdad en el ingreso tiene un fuerte efecto negativo sobre la EVN, y que esta relación puede estar mediada por el capital social, expresado en los niveles de corrupción y las diferencias culturales. Estos resultados son consistentes con los observados en otros estudios $(21,24,25)$, aunque deben leerse con cautela ya que existen algunas aproximaciones teóricas que indican que la desigualdad en el ingreso y el capital social pueden afectar la salud por diferentes mecanismos causales, siendo ambos dependientes de otras formas de desigualdad que también actúan en los niveles de agregación más elevados (26-30). Lo interesante para el caso mexicano, es que el efecto de la desigualdad en el ingreso se mantiene sin importar el mecanismo, aunque disminuye su magnitud al actuar directamente sobre la EVN y ajustarse por los otros macro-determinantes.

El haber usado variables ecológicas y explorar inferencias únicamente en ese nivel evita que se tenga una falacia ecológica. La no inclusión de otros macro-determinantes en los modelos múltiples puede resultar en que el efecto observado esté confundido; infortunadamente, aún falta mucho desarrollo teórico que permita señalar cuál o cuáles son estas variables, lo cual resulta más complejo debido a la ausencia de datos disponibles para tal fin. Algo similar ocurre si se quieren hacer inferencias a nivel individual, ya que la relación observada puede estar confundida por variables de nivel inferior al de entidad federativa (31).

Hasta donde sabemos, este es el primer estudio en epidemiología social que utiliza la corrupción ajustada por diferencias culturales, expresadas por la religión y el indigenismo, como proxy del capital social. Tanto el legado indígena como la religión católica son atributos importantes de la cultura mexicana (32). Innovaciones como esta son importantes, ya que la selección de indicadores no debe seguir una "receta" única sino debe considerar los modelos teóricos propios de cada contexto de investigación (33); el observar resultados consistentes pese a usar diferentes indicadores lo que hace es fortalecer los conceptos teóricos subyacentes. Sin embargo, otros estudios podrán explorar otros indicadores de capital social y sus posibles mecanismos de acción. En conclusión, este estudio aporta evidencias de que en México la desigualdad en el ingreso es un poderoso macro-determinante de la salud poblacional que actúa por un mecanismo que involucra el capital social y las diferentes formas de discriminación. Futuros estudios que incorporen variables de niveles de agregación inferior podrán explorar si este efecto también se 
REVISTA DE SALUD PÚBLICA • Volumen 7 (2), Julio 2005

observa en la salud individual de los mexicanos, tal como se ha hecho en otras regiones (34) *

\section{REFERENCIAS}

1. Marmot MG. Understanding social inequalities in health. Perspect. Biol. Med 2003; 46(supl.3):S9-S23.

2. Rodgers GG. Income and inequality as determinants of mortality: An international cross-section analysis. Pop. Stud 1979; 33:343-351.

3. Wilkinson RG. Income distribution and life expectancy. Br. Med. J 1992; 304:165-168.

4. Kennedy BP, Kawachi I, Glass R, Prothrow-Stith D. Income distribution, socioeconomic status, and self rated health in the United States: Multilevel analysis. Br. Med. J 1998; 317:917-921.

5. Subramanian SV, Kawachi I. Income inequality and health: What have we learned so far? Epidemiol. Rev 2004; 26:78-91.

6. Lochner K, Kawachi I, Kennedy BP. Social capital: A guide to its measurement. Health Place 1999; 5:259-270.

7. Kawachi I, Berkman L. Social cohesion, social capital, and health. In: Berkman LF, Kawachi I (ed.). Oxford: Oxford University Press; 2000. p. 174.

8. Bjørnskov C. The happy few. Cross-country evidence on social capital and life satisfaction. Kyklos 2003; 56:3-16.

9. Putnam RD. Making democracy work. Princeton: University Press; 1993.

10. Narayan D, Cassidy MF. A dimensional approach to measuring social capital: Development and validation of a social capital inventory. Curr. Sociol 2001; 49:59-105.

11. Sampson RJ, Groves WB. Community structure and crime: Testing social disorganization theory. Am. J. Sociol 1989; 94:774-802.

12. Saegert S, Winke G. Crime, social capital, and community participation. Am. J. Community Psychol 2004; 34:219-233.

13. McMullan M. A theory of corruption. Soc. Rev 1961; 9:181-201.

14. Boltvinik J. La pobreza en México. II. Magnitud. Salud Publica Mex 1995; 37:298-309.

15. Paldam M. Corruption and religion adding to the economic model. Kyklos 2001; 54:383-414.

16. McCann JC. A technique for estimating life expectancy with crude vital rates. Demography 1976;13:259-272.

17. Arriaga EE. Measuring and explaining the change in life expectancies. Demography 1984; 21:83-96.

18. Tuirán-Gutiérrez R. La distribución del ingreso monetario en México. Este País; 2003. p. 142.

19. Haidich AB, Ioannidis JP. The Gini coefficient as a measure for understanding accrual inequalities in multicenter clinical studies. J. Clin. Epidemiol 2004; 57:341-348. 
20. Transparencia Mexicana. Encuesta Nacional de Corrupción y Buen Gobierno 2001 [Internet] Disponible en: http://www.transparenciamexicana.org.mx. Acceso: 15 de febrero de 2005.

21. Kawachi I, Kennedy BP, Lochner K, Prothrow-Stith D. Social capital, income inequality, and mortality. Am. J. Public Health 1997; 87:1491-1498.

22. Newhouse JP, McClellan M. Econometrics in outcomes research: The use of instrumental variables. Annu. Rev. Public Health 1998; 19:17-34.

23. Hausman J. Specification tests in econometrics. Econometrica 1978; 46:12511271.

24. Lynch JW, Kaplan GA, Pamuk ER, Cohen RD, Heck KE, Balfour JL, Yen IH. Income inequality and mortality in metropolitan areas of the United States. Am. J. Public Health 1998; 88:1074-1080.

25. Lynch J, Smith GD, Hillemeier M, Shaw M, Raghunathan T, Kaplan G. Income inequality, the psychosocial environment, and health: Comparisons of wealthy nations. Lancet 2001; 358:194-200.

26. Muntaner C, Lynch J. Income inequality, social cohesion, and class relations: A critique of Wilkinson's neo-Durkheimian research program. Int. J. Health Serv 1999; 29:59-81.

27. Navarro V, Shi L. The political context of social inequalities and health. Soc. Sci. Med 2001; 52:481-491.

28. Braveman P, Tarimo E. Social inequalities in health within countries: Not only an issue for affluent nations. Soc. Sci. Med 2002; 54:1621-1635.

29. Deaton A. Health, inequality, and economic development. J. Econ. Lit 2003; 41:113-158.

30. Coburn D. Beyond the income inequality hypothesis: Class, neo-liberalism, and health inequalities. Soc. Sci. Med 2004; 58:41-56.

31. Greenland S. Ecologic versus individual-level sources of bias in ecologic estimates of contextual health effects. Int. J. Epidemiol 2001; 30:1343-1350.

32. Merrell F. The Mexicans: A sense of culture. Oxford: Westview Press; 2003.

33.Kawachi I, Kennedy BP. The relationship of income inequality to mortality: Does the choice of indicator matter? Soc. Sci. Med 1997; 45:1121-1127.

34. Blakely TA, Lochner K, Kawachi I. Metropolitan area income inequality and self-rated health - a multilevel study. Soc. Sci. Med 2002; 54:65-77. 
